\title{
Assessing heat exchanger performance data using temperature measurement uncertainty
}

\author{
R.A. Tatara ${ }^{1}$, G.M. Lupia ${ }^{2}$ \\ ${ }^{1 *}$ Department of Technology, Northern Illinois University, USA \\ ${ }^{2}$ CSI Technologies, Inc., Elgin, Illinois, USA \\ "Corresponding Author: e-mail: tatara@ceet.niu.edu, Tel +1-815-753-1130,Fax.+1-815-753-3702
}

\begin{abstract}
To ensure operation of heat exchangers, the goal is to verify that the exchanger is performing, or will perform, at its design. This is especially relevant to coolers that typically operate at heat loads reduced from their design basis. In addition, any calculated performance acceptance criteria must also consider uncertainty and error in the experimental measurements of temperature and flow. However, most statistical methods are complex and not easily applied to heat exchangers such as those that serve the power plant industry where data are difficult to obtain and limited in quantity. To address this, a concise, practical, and effective methodology has been formulated, with its emphasis on temperature measurement, based on a Student tdistribution with a 95\% confidence level. A review of temperature instrumentation has included expected accuracies of various measurement techniques. Detailed calculations illustrate the significant effect of decreasing the sampling frequency; reducing the number of sensors especially increases the uncertainty level. An $11.6 \%$ overall heat load uncertainty due to flow and temperature measurements was computed for a sample water-to-water cooler using steady-state field data. Results were consistent with those of other research efforts.
\end{abstract}

Keywords: Heat exchanger, uncertainty analysis, temperature measurement, shell-and-tube.

DOI: http://dx.doi.org/10.4314/ijest.v3i8.1

\section{Introduction}

The goal of heat exchanger performance monitoring is to ensure that an exchanger is performing, or will perform, at its design basis. It is especially important for cooling system heat exchangers in the nuclear power plant industry that must meet their design basis which may be at much higher heat loads than their standard operating conditions. Assuming all other system components are functioning properly, it is fouling or flow blockage that would reduce the heat transfer capability of an exchanger below its design limits; any performance monitoring program must identify a decrease in the overall heat transfer coefficient. There are a variety of monitoring and testing methods used in the nuclear industry but the only true test method is one which directly addresses the heat transfer capability.

If the true design flows, heat load, and temperatures cannot be achieved in testing, the test data must be extrapolated (taking into account tube plugging) to compute the overall heat transfer coefficient and compare it to the design value. But any calculation must also consider uncertainty and error in the experimental measurements of temperature and flow. Furthermore, even before an overall coefficient is computed, the test data must be assessed to have sufficient accuracy. This may be done as a pretest where experimental parameters are estimated, and it is judged whether the overall heat transfer coefficient can be meaningfully found.

For power plant personnel such analysis is challenging due to the fact that information must be drawn from instrument design and operation, heat transfer applications, and statistical theory. Certainly a great amount of published work already deals with these topics. Although statistical methods are numerous, many techniques are cumbersome and difficult to apply to actual power plant heat exchangers as measurements from operating units are often limited in sample size and quality; plant personnel must have a relatively simple and practical, but effective, method to judge the data. However, a simplified methodology specific to power plant coolers is not readily available in the common literature. Knowledge of instrument sensor accuracy, impact of number 
of sensors, data sampling intervals, and a reasonable statistical confidence level are some of the factors to be considered, especially in the case of temperature measurement. To address this, the present work, drawing from industry standards, presents a centralized, practical statistical approach and quantifies these factors so that heat exchanger temperature data may be easily and quickly assessed. Furthermore, sample calculations are presented to facilitate the use of the proposed methodology. Although the concentration is on temperature measurement, the procedure can be easily extended to flow measurement or any other parameter.

\section{Literature Survey}

From a theoretical, design standpoint, it is often assumed that there are no variations (uncertainties) in parameters such as temperatures, local heat transfer coefficients, tube wall thicknesses, and fouling factors. As compensation, conservative values are selected or some arbitrary excess heat transfer area is included in the design. A more quantitative approach to this problem was formulated by Cho (1987) utilizing a Gaussian form of uncertainty distribution for the key parameters of local heat transfer coefficients and tube wall thickness to compute excess shell-and-tube exchanger area required to ensure design performance. A sample case indicated that $6.3 \%$ and $20 \%$ excess areas ensured performance at $80 \%$ and $99 \%$ confidence levels, respectively. Extending this work by considering uncertainty distributions other than Gaussian, a Monte Carlo simulation was used to determine the additional area a heat exchanger must have to compensate for uncertainties in theoretical heat transfer coefficients, tube wall thermal conductivity, and tube diameters (Badar et al., 1993). A sample analysis showed that $7 \%$ and $30 \%$ additional heat transfer areas would be needed to achieve $80 \%$ and $99 \%$ confidence levels, respectively. Additionally, Prasad et al. (2002) quantified the effect of uncertainty in local heat transfer coefficients and inlet temperatures upon the overall performance of a tube-in-tube exchanger. The relative contribution of all input parameters upon the overall uncertainty for a compact heat exchanger was found by James et al. (1995).

Less information is available in the common literature involving in-service heat exchangers with actual performance data. Lewis and Phillips (1993) addressed bias and precision errors in evaluating data from water-cooled exchangers in nuclear power plants. Their computations demonstrated that fouling factors cannot be accurately determined without careful consideration of instrument and measurement uncertainties; and in an extreme case more than $900 \%$ error in the fouling factor was encountered due to small temperature differences and the use of only a single sensor at each temperature measurement location.

2.1 Temperature measurement in heat exchangers: Many different types of temperature-measuring instruments exist. For heat exchanger performance monitoring, instruments and probes based on electrical effects are most common; specifically, RTDs, thermistors, and thermocouples are used.

Electrical resistance thermometers (RTDs) operate on the principle that a metal's electrical resistance changes with temperature. RTDs have typical uncertainties/errors of $0.1{ }^{\circ} \mathrm{C}$ although special ones can give plus or minus $0.01{ }^{\circ} \mathrm{C}$ and can be used to calibrate other instruments such as thermocouples. Generally, RTDs are stable but relatively bulky and fragile. Slower response times and self-heating errors (since RTDs require a current supply which can heat the sensor itself) are other drawbacks. Without special calibration, industrial-grade probes have uncertainties ranging from approximately $0.06{ }^{\circ} \mathrm{C}$ to $0.6{ }^{\circ} \mathrm{C}$ (EPRI, 1998).

Thermistors are sintered, ceramic semiconductor devices utilizing a non-linear temperature coefficient of resistance. These are very sensitive to small temperature changes and have a high degree of accuracy $\left(+/-0.01{ }^{\circ} \mathrm{C}\right)$. The thermistor is limited to narrow temperature ranges due to the non-linear nature of the temperature versus resistance relation. Generally, uncertainties are $0.06{ }^{\circ} \mathrm{C}$ to $0.6{ }^{\circ} \mathrm{C}$ (EPRI, 1998). Probes can be made very small and exhibit fast response to temperature changes. However, such small probes would be more affected by self-heating errors, similar to RTDs, and are rather fragile.

The basis for thermocouple measurement is the thermoelectric effect produced when two dissimilar metals are mechanically joined and a circuit voltage (Seebeck voltage) is produced. The voltage depends on the metal junction temperature and the types of metals joined. Multiple thermocouples (TCs) can be connected in series to form a thermopile, essentially an averaging of the individual readings at the same location. A thermocouple is rugged and convenient but must be properly installed and may degrade over time depending on its materials; iron-based thermocouples are susceptible to rusting. Without special calibrations, thermocouple uncertainties range from $0.6{ }^{\circ} \mathrm{C}$ to $2.8^{\circ} \mathrm{C}$ (EPRI, 1998). When the optimal thermocouple probe is carefully calibrated and correctly installed, $+/-0.1{ }^{\circ} \mathrm{C}$ is achievable although an uncertainty of $+/-0.6{ }^{\circ} \mathrm{C}$ is a more reasonable goal for a standard industrial application. Uncertainties stem from variations in thermocouple materials; Peltier, Thomson, and Joule effects; thermocouple junction and probe construction; heat conduction within the probe; and distortion of the temperature field due to the presence of the probe itself, among others.

Any temperature probe must be correctly installed and a variety of probe configurations and installation standards exist for wells, flat surfaces, curved pipes, and high-temperature environments. For example, due to the heat transfer process that exists when the fluid temperature is not identical to the surrounding air (ambient), surface temperature measurement errors are present unless the temperature sensor or probe is sufficiently isolated from the ambient. These are commonly referred to as backside heat losses, and it is desirable to minimize such losses. The proper installation is specified by standards (ASME, 1974); a typical standard will detail the pipe wall preparation (cleaning); the attachment of the thermocouple/RTD probe to the pipe wall; the type of temperature probe (flat, contoured, twisted wire bead, etc.); the addition of thermal paste, grease, or epoxy to ensure good probe-to-metal contact; type of insulation covering the probe (closed cell elastomeric, fiberglass, etc.); the thickness of insulation; and taping and 
securing the insulation bundle to the outside of the pipe. Thermowell installations are also adequately described (EPRI, 1998, ASME, 2010). In any case, the geometry of the probe and well; use of thermal grease to improve thermal contact and eliminate trapped air; immersion depth; and backside heat losses are factored into the measurement process.

2.2 Data recording: Typically for water-to-water heat exchangers, inlet and outlet fluid temperatures are recorded and monitored. Data must meet steady-state and differential temperature requirements; generally, at least ten minutes of steady-state data are required. As a minimum it is recommended to measure each temperature parameter (hot side inlet, hot side outlet, cold side inlet, and cold side outlet) at three or, preferably, four locations. Multiple measurements of each parameter will minimize any effects of thermal streaming and instrument inaccuracy and provide assurance in the event one instrument fails. In terms of probability, the confidence level is increased with multiple measurements as will be shown.

As steady-state measurements are the most useful, a criterion is needed to ensure steady conditions during data logging by manual means or with a data acquisition system (DAS). The Electric Power Research Institute (EPRI, 1998) suggests a $0.02{ }^{\circ} \mathrm{C}$ per minute stability for a heat exchanger inlet fluid temperature. Individual RTD or thermocouple readings may deviate from this guideline but the mean (average value) would still be within the criterion. This is acceptable considering that the heat transfer calculations are based upon means. A reasonable data collection procedure may specify the recording of data at 1-minute intervals over a 30-minute test time. (This may be altered should the residence time of either fluid be exceptionally large or small; the size of the heat exchanger and the fluid flow rates will affect the residence times.) If the 1-minute interval is strictly maintained, a wellaveraged value can be obtained from the data points for each temperature.

\section{Uncertainty Analysis Procedure}

Much of statistical analysis is based on large-sample data sets and a normal (Gaussian) error distribution. For this approach, the probability, or confidence, that an individual data point, such as a temperature measurement, will fall within an expected range is easily computed from the normal-distribution function. The expected range is given in terms of one, two, three, etc., standard deviations from the mean value; a higher standard deviation, $\sigma$, indicates that more data points will fall within the expected range. For a normal distribution:

$$
\sigma=\left[\frac{\sum_{i=1}^{N}\left(X_{i}-X_{m}\right)^{2}}{N}\right]^{1 / 2}
$$

where $X_{i}$ is a single data point or measurement, $X_{m}$ is the mean of single measurements, and $N$ is the number of measurements.

But the standard deviation of Eq. (1) is theoretical in that it assumes an infinite number of measurements from an individual sensor such as a thermocouple. In practice, the best estimate for the standard deviation in a set of $N$ measurements is the unbiased or sample standard deviation, $S_{x}$, defined by Eq. (2).

$$
S_{x}=\left[\frac{\sum_{i=1}^{N}\left(X_{i}-X_{m}\right)^{2}}{N-1}\right]^{1 / 2}
$$

This represents a better estimate of the deviation of an individual measurement from the mean of all the data points. Often in heat exchanger temperature measurement, multiple probes or instruments are used to sample the same parameter such as an inlet or outlet fluid temperature. In this case, measurements are averaged and the interest is in the deviation of the mean temperature. Eq. (3) presents the expected deviation of the mean of readings, $S_{m}$, from the true mean.

$$
S_{m}=\left[\frac{\sum_{i=1}^{N}\left(X_{i}-X_{m}\right)^{2}}{N(N-1)}\right]^{1 / 2}
$$

Or,

$$
S_{m}=S_{x} / N^{1 / 2}
$$


Comparing Eqs. (2) and (3), it is clear that the deviation of a mean temperature reading will be less than the deviation of a single data point $\left(S_{m}<S_{x}\right)$; this illustrates the advantage of using mean values. These mean values can be taken over time while maintaining steady-state conditions. However, if using only one temperature sensor, there is the possibility of bias introduced to the data, no matter how many data points are collected. A better approach would be to use multiple probes to measure the same parameter. Temperature readings now can be averaged over several probes as well as time. This will serve to decrease $S_{m}$ as well as guard against instrument bias.

3.1 The Student t-distribution: However, these statistical methodologies for averaged data are predicated upon Gaussian- or normal-error-distributed data. It is well-known that sampling sizes smaller than about 20-30 are not suitable for normal-error analysis and are often better correlated by the Student t-distribution. The t-distribution is also based on symmetrical and bellshaped curves with a mean of zero and standard deviations from the mean. The general shape of the curve is dependent on the number of degrees of freedom. Student t-distributions are a function of degrees of freedom and percent confidence level. A confidence level is an alternate expression for standard deviation and is commonly presented as 50, 80, 90, 95, 99, and 99.9 percent intervals. For instance, with an infinitely large data set, the $90 \%$ confidence level corresponds to $+/-1.645 \sigma$, the $95 \%$ level equals $+/-1.960 \sigma$, and $99.9 \%$ corresponds to $+/-3.291 \sigma$. A confidence level is the probability that a mean measurement lies within a certain amount of standard deviations. The uncertainty ranges increase as the number of data (degrees of freedom) decrease. The t-distribution method is more accurate for the smaller data sets encountered in heat exchanger testing and is computed by Eq. (5).

$$
S_{m t}=t_{\nu-\%} S_{x} / N^{1 / 2} \quad \text { or } \quad S_{m t}=t_{\nu-\%} S_{m}
$$

The t-distribution theory and tabular values, for various combinations of degrees of freedom $(v)$ and percent confidence levels, are readily available (Coleman and Steele, 1999).

3.2 The "true" temperature: With power plant heat exchangers, EPRI (1998) has recommended for calculations or assessments that the true measurement of a parameter such as temperature be determined from space-averaged (spatial) and time-averaged (temporal) measurements. Furthermore, each individual measurement is considered to be independent of position and time; thus each individual data point is given equal weight. This allows for multiple probes measuring the same parameter. An example would be several thermocouples located around the circumference of a pipe; furthermore, data could be obtained over several minutes. Both spatial and temporal averaging would take place to produce the "true" value for a fluid temperature.

From statistical analysis, it is apparent that the numbers of spatial and temporal data points contribute to the overall uncertainty in temperature measurement. The uncertainty is a function of the number of temperature probes and the number of total data points obtained. These contributions must be calculated and taken into account along with any inherent error or bias in the instrumentation, data acquisition, installation, or in the probe itself; a 95\% t-distribution confidence level is recommended when assessing the relative contribution of each deviation source (EPRI, 1998).

For an application of this statistical approach with plant data with multiple resistance thermometer elements (RTDs), EPRI (1998) has data for the cold fluid inlet temperature of an operating heat exchanger. The following sample calculation displays the effect on uncertainty when the number of RTDs and the sampling time interval are varied. Three RTDs are located at the inlet while data are sampled every five minutes up to a twenty-minute span. Individual temperature readings are presented in Table 1.

Table 1. Individual temperature data for sample calculation

\begin{tabular}{|c|c|c|c|}
\hline $\begin{array}{c}\text { Time } \\
(\mathrm{min})\end{array}$ & $\begin{array}{c}\mathrm{T} \# 1 \\
\left({ }^{\circ} \mathrm{C}\right)\end{array}$ & $\begin{array}{c}\mathrm{T} \# 2 \\
\left({ }^{\circ} \mathrm{C}\right)\end{array}$ & $\begin{array}{c}\mathrm{T} \# 3 \\
\left({ }^{\circ} \mathrm{C}\right)\end{array}$ \\
\hline 5 & 30.15 & 30.10 & 30.12 \\
\hline 10 & 30.20 & 30.11 & 30.10 \\
\hline 15 & 30.22 & 30.16 & 30.15 \\
\hline 20 & 30.18 & 30.17 & 30.16 \\
\hline
\end{tabular}

Since each individual data point of Table 1 is considered valid, an arithmetic mean of the twelve values is calculated by:

$$
X_{m}=\frac{\sum_{i=1}^{N} X_{i}}{N}=\frac{30.15+30.10+30.12+\ldots+30.18+30.17+30.16}{12}=30.152{ }^{\circ} \mathrm{C}
$$

Thus $30.152{ }^{\circ} \mathrm{C}$ is considered the true cold fluid inlet temperature. But this value must be assessed in terms of uncertainties in space, time, and instrument. Although the data are obtained at steady-state and the sampling interval is assumed to be longer than the sampling response time, it is necessary to evaluate the temporal uncertainty in the data. Accordingly, the mean of all RTDs is 
$30.123,30.137,30.177$, and $30.170{ }^{\circ} \mathrm{C}$ for sampling times $5,10,15$, and 20 minutes, respectively. It must be determined whether this time averaging has introduced significant deviation in the true temperature reading of $30.152^{\circ} \mathrm{C}$. Since this involves deviation between means, Eq. (3) is used.

$$
S_{m}=\left[\frac{(30.123-30.152)^{2}+(30.137-30.152)^{2}+(30.177-30.152)^{2}+(30.170-30.152)^{2}}{4(4-1)}\right]^{1 / 2}=0.0130{ }^{\circ} \mathrm{C}
$$

Assessing this uncertainty in terms of the 95\% t-distribution confidence and N-1 (4-1=3) degrees of freedom,

$$
S_{m(\text { temporal })}=t_{3-95 \%} S_{m}=3.182\left(0.0130{ }^{\circ} \mathrm{C}\right)=0.0414^{\circ} \mathrm{C}
$$

To determine the effects of shortening the sampling time, the data set is reduced from 20 minutes to 15 minutes so that the total data from Table 1 are restated in Table 2 up to only 15 minutes along with each mean RTD reading. For these nine temperatures, the total mean is $30.146{ }^{\circ} \mathrm{C}$ and considered the true cold fluid inlet temperature for this data set. As before, it must be determined whether time averaging has introduced significant deviation from the true temperature reading of $30.146{ }^{\circ} \mathrm{C}$. Again, Eq. (3) is used,

$$
S_{m}=\left[\frac{(30.123-30.146)^{2}+(30.137-30.146)^{2}+(30.177-30.146)^{2}}{3(3-1)}\right]^{1 / 2}=0.0162{ }^{\circ} \mathrm{C}
$$

while for 3-1=2 degrees of freedom:

$$
S_{m(\text { temporal })}=t_{2-95 \%} S_{m}=4.303\left(0.0162{ }^{\circ} \mathrm{C}\right)=0.0697{ }^{\circ} \mathrm{C}
$$

This represents a $68 \%$ increase in uncertainty level compared to the 20 -minute sampling calculated previously. Clearly temporal sampling effects are significant.

Table 2. Individual temperature data for a 15-minute sampling

\begin{tabular}{|c|c|c|c|c|}
\hline $\begin{array}{c}\text { Time } \\
(\min )\end{array}$ & $\begin{array}{c}\mathrm{T} \# 1 \\
\left({ }^{\circ} \mathrm{C}\right)\end{array}$ & $\begin{array}{c}\mathrm{T} \# 2 \\
\left({ }^{\circ} \mathrm{C}\right)\end{array}$ & $\begin{array}{c}\mathrm{T} \# 3 \\
\left({ }^{\circ} \mathrm{C}\right)\end{array}$ & $\begin{array}{c}(\mathrm{T} \# 1+\# 2+\# 3) / 3 \\
\left({ }^{\circ} \mathrm{C}\right)\end{array}$ \\
\hline 5 & 30.15 & 30.10 & 30.12 & 30.123 \\
\hline 10 & 30.20 & 30.11 & 30.10 & 30.137 \\
\hline 15 & 30.22 & 30.16 & 30.15 & 30.177 \\
\hline
\end{tabular}

Supplemental to temporal effects, it must be determined whether spatial averaging also has introduced comparable bias to the true temperature reading of $30.152{ }^{\circ} \mathrm{C}$. Again, since means are compared, Eq. (3) applies with spatial-averaged temperatures of $30.188,30.135$, and $30.133^{\circ} \mathrm{C}$, for $\mathrm{T} \# 1, \mathrm{~T} \# 2$, and $\mathrm{T} \# 3$, respectively.

$$
S_{m(\text { spatial })}=\left[\frac{(30.188-30.152)^{2}+(30.135-30.152)^{2}+(30.133-30.152)^{2}}{3(3-1)}\right]^{1 / 2}=0.0180{ }^{\circ} \mathrm{C}
$$

This deviation may be converted to a bias based on the $95 \%$ confidence level from a t-distribution of Eq. (5):

$$
B_{m(\text { spatial })}=t_{2-95 \%} S_{m(\text { spatial })}
$$

For three minus one degrees of freedom and the $95 \%$ confidence, $t_{2-95 \%}=4.303$ so that the bias due to spatial uncertainty is:

$$
B_{m(\text { spatial })}=4.303\left(0.0180{ }^{\circ} \mathrm{C}\right)=0.0775^{\circ} \mathrm{C}
$$

The advantage of multiple measurement locations to reduce sample uncertainty can be demonstrated by recalculating this spatial uncertainty with data using only two probes: $\mathrm{T} \# 1$ and $\mathrm{T} \# 2$. The mean of these eight data points from Table 3 is $30.162{ }^{\circ} \mathrm{C}$ and the spatial bias is once more computed from Eq. (3): 


$$
S_{m(\text { spatial })}=\left[\frac{(30.188-30.162)^{2}+(30.135-30.162)^{2}}{2(2-1)}\right]^{1 / 2}=0.0265{ }^{\circ} \mathrm{C}
$$

Again, this deviation may be converted to a bias based on the $95 \%$ confidence level from a t-distribution with Eq. (5). For two minus one degrees of freedom and the $95 \%$ confidence, $t_{1-95 \%}=12.706$ so that the bias due to spatial uncertainty by using only two RTDs is:

$$
B_{m(\text { spatial })}=t_{1-95 \% \circ} S_{m(\text { spatial })}=12.706\left(0.0265^{\circ} \mathrm{C}\right)=0.3367^{\circ} \mathrm{C}
$$

This is significantly higher, compared to $0.0775{ }^{\circ} \mathrm{C}$, and illustrates the value of multiple probes, including the addition of just one more probe. Table 4 summarizes the results which illustrate the effects of time-averaging and adding more RTDs and indicates substantial increase in temperature uncertainty as the time interval (number of data samplings) and available RTDs are reduced.

Table 3. Individual temperature data using only two probes

\begin{tabular}{|c|c|c|}
\hline $\begin{array}{c}\text { Time } \\
(\mathrm{min})\end{array}$ & $\begin{array}{c}\mathrm{T} \# 1 \\
\left({ }^{\circ} \mathrm{C}\right)\end{array}$ & $\begin{array}{c}\mathrm{T} \# 2 \\
\left({ }^{\circ} \mathrm{C}\right)\end{array}$ \\
\hline 5 & 30.15 & 30.10 \\
\hline 10 & 30.20 & 30.11 \\
\hline 15 & 30.22 & 30.16 \\
\hline 20 & 30.18 & 30.17 \\
\hline mean $(5-20)$ & 30.188 & 30.135 \\
\hline
\end{tabular}

Table 4. Effects upon temporal and spatial uncertainties by varying sampling time and number of RTDs

\begin{tabular}{|c|c|c|c|}
\hline Number of RTDs & Number of 5-minute samplings & $\begin{array}{c}\text { Uncertainty } \\
\left({ }^{\circ} \mathrm{C}\right)\end{array}$ & Uncertainty type \\
\hline 3 & 4 & 0.0414 & temporal \\
\hline 3 & 3 & 0.0697 & temporal \\
\hline 3 & 4 & 0.0775 & spatial \\
\hline 2 & 4 & 0.3367 & spatial \\
\hline
\end{tabular}

3.3 Combining temperature uncertainties: It remains to combine and total all possible uncertainties. At this point a review of the uncertainty types is helpful. Uncertainties may be classified as one of two types: random or predictable (systematic). Random errors are precision deviations and are unpredictable and may come from different sources. One common example of this type of uncertainty is the routine scatter of data during measurements. Other examples include temporal variations, environmental fluctuations (such as ambient temperature changes affecting temperature probe readings), signal noises, and other random effects. It may be possible to compute the uncertainty due to any or all of these in terms of standard deviation, $S_{m}$, as was done with the time-averaged data in the sample calculation.

On the other hand, a predictable uncertainty is better termed bias. Examples include spatial effects of the type assessed in the sample calculation, instrument calibration errors, data acquisition errors, and others that are expected and anticipated. Not all of these would be statistically quantifiable but could include judgment. For instance, the overall accuracy of a DAS unit may be reported by the manufacturer as $+/-1.1{ }^{\circ} \mathrm{C}$. This would be the bias deviation, $B_{m}$, and could not be statistically verified by the user of the unit during temperature data acquisition. Thus combining all uncertainties (EPRI, 1998):

$$
U_{T}=\left[B_{T}^{2}+\left(t_{v-95 \%} S_{T}\right)^{2}\right]^{1 / 2}
$$

where $U_{T}$ is the overall uncertainty in true temperature at $95 \%$ confidence, $B_{T}$ is the total bias deviations for temperature, and $t_{v-95 \%} S_{T}$ is the total standard deviations at $95 \%$ confidence. In essence the total bias $\left(B_{T}\right)$ is the probable, expected, or predicted offset from the true temperature while the standard deviation term $\left(S_{T}\right)$ represents the total random scatter of data points around that offset. Either term may be relatively large or small compared to the other one. This is illustrated in Figure 1 which displays a measurement containing a large amount of bias and a rather narrow, limited range of random scatter. Assuming all the biases are independent from each other, or that one measurement does not impact another,

$$
B_{T}=\left[\sum_{i=1}^{J}\left(\theta_{i} B_{i}\right)^{2}\right]^{1 / 2}
$$


Eq. (7) represents the total bias where $\theta_{i}$ is the sensitivity coefficient for the $i$ th parameter and $B_{i}$ is its bias. In the sample case of the cold fluid inlet temperature, its measurement does not depend on any other parameter so that $\theta_{1}=\theta_{2}=\ldots=\theta_{i}=\partial T / \partial T=1$.

To illustrate the use of Eqs. (6) and (7), the spatial deviation from the data set of Table 1 is added to an additional probe/DAS bias of $0.5556{ }^{\circ} \mathrm{C}$. Thus, the total bias considers the RTD probe/DAS deviation along with the previously-computed spatial deviation:

$$
B_{T}=\left[\sum_{i=1}^{J}\left(\theta_{i} B_{i}\right)^{2}\right]^{1 / 2}=\left[\left[(1)\left(0.5556{ }^{\circ} \mathrm{C}\right)\right]^{2}+\left[(1)\left(0.0775^{\circ} \mathrm{C}\right)\right]^{2}\right]^{1 / 2}=0.5610{ }^{\circ} \mathrm{C}
$$

Note that the $95 \%$ confidence limit is already contained within each individual bias term, where applicable. The bias due to spatial averaging has been adjusted for this confidence level; the DAS bias of $0.5556{ }^{\circ} \mathrm{C}$ is unadjusted because it is a manufacturer's bias without multiple data points for statistical evaluation. This is why in Eq. (6) $B_{T}$ is unadjusted while $S_{T}$ is multiplied by $t_{v-95 \%}$. Next, the random uncertainty contribution is computed under the assumption that the only source is due to the previously-computed temporal averaging, or

$$
S_{T}=\left[\sum_{i=1}^{J}\left(\theta_{i} S_{i}\right)^{2}\right]^{1 / 2}=\left[(1)\left(0.0130{ }^{\circ} \mathrm{C}\right)^{2}\right]^{1 / 2}=0.0130{ }^{\circ} \mathrm{C}
$$

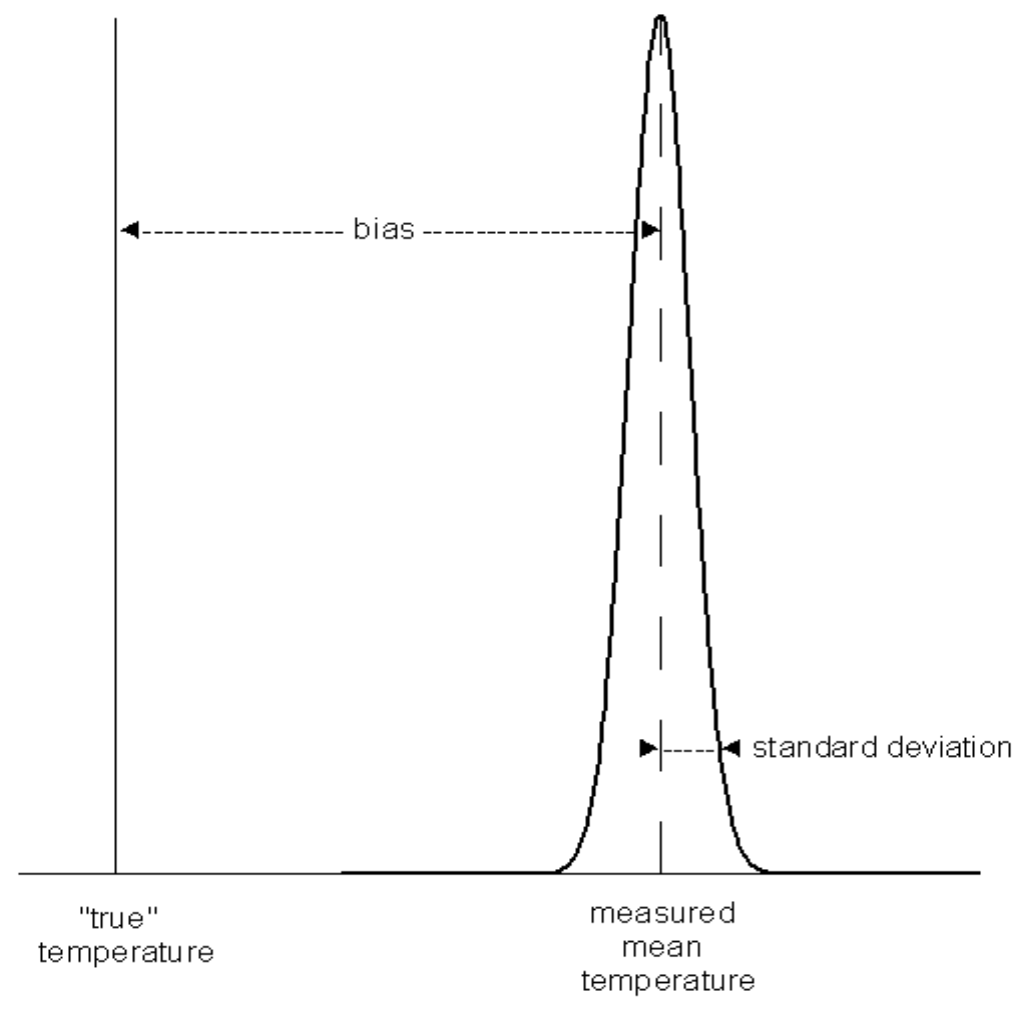

Figure 1. Temperature measurement probability distribution having a large bias and a small scatter

Finally, combining all the uncertainties:

$$
U_{T}=\left[B_{T}^{2}+\left(t_{3-95 \%} S_{T}\right)^{2}\right]^{1 / 2}=\left[\left(0.5610{ }^{\circ} \mathrm{C}\right)^{2}+\left[(3.182)\left(0.0130{ }^{\circ} \mathrm{C}\right)\right]^{2}\right]^{1 / 2}=0.5625^{\circ} \mathrm{C}
$$

Note that here the t-distribution degrees of freedom are identical to the value used in the temporal deviation computations. This is possible because only one contribution to $S_{T}$ exists. When multiple sources contribute to the total random data variance, some 
weighted-average degrees of freedom must be calculated to obtain the t-distribution value in Eq. (6). Such calculations are beyond the scope of this study and are detailed elsewhere (EPRI, 1998) and take into account sensitivity coefficients and individual degrees of freedom from each source of uncertainty. Additionally, if measurements are not completely independent of each other, then correlation coefficients must be found to assess the dependency among uncertainty sources.

\section{Results and Discussion}

Once temperature - and other - uncertainties are assessed, it is necessary to evaluate the heat load calculations. A methodology has been tailored to operating power plant exchangers and coolers by EPRI (1998). A heat load determination may be made from one or both sides of the heat exchanger. For the hot fluid side,

and for the cold fluid side,

$$
Q_{h}=m_{h} \times c p_{h} \times\left(T_{h I}-T_{h O}\right)
$$

$$
Q_{c}=m_{c} \times c p_{c} \times\left(T_{c O}-T_{c I}\right)
$$

As a function of measurement accuracy, an error analysis must be made on the heat duty for each side of the heat exchanger by a propagation-of-error method (Coleman and Steele, 1999) to compute an uncertainty level for heat load. Theoretically, it is desired to compute the uncertainty, $U_{R}$, in any parameter $R$ where $R$ is a function of independent variables such that $R=f\left(Z_{l}, Z_{2}, Z_{3}, \ldots Z_{n}\right)$ and $U_{1}, U_{2}, U_{3}, \ldots U_{n}$ are the uncertainties in the individual parameter. The result is:

$$
U_{R}=\sqrt{\left[\left(\frac{\partial R}{\partial Z_{1}}\right) \times U_{1}\right]^{2}+\left[\left(\frac{\partial R}{\partial Z_{2}}\right) \times U_{2}\right]^{2}+\left[\left(\frac{\partial R}{\partial Z_{3}}\right) \times U_{3}\right]^{2}+\ldots+\left[\left(\frac{\partial R}{\partial Z_{n}}\right) \times U_{n}\right]^{2}}
$$

As an example, for the hot or cold side fluid heat load, $Q$,

$$
U_{Q}=\sqrt{\left[\left(\frac{\partial Q}{\partial m}\right) \times U_{m}\right]^{2}+\left[\left(\frac{\partial Q}{\partial c p}\right) \times U_{c p}\right]^{2}+\left[\left(\frac{\partial Q}{\partial T_{I}}\right) \times U_{T_{I}}\right]^{2}+\left[\left(\frac{\partial Q}{\partial T_{O}}\right) \times U_{T_{O}}\right]^{2}}
$$

The individual partial derivative terms may be evaluated. The flow contribution to uncertainty is $\frac{\partial Q}{\partial m}=c p \times\left(T_{I}-T_{O}\right)$; the specific heat contribution is $\frac{\partial Q}{\partial c p}=m \times\left(T_{I}-T_{O}\right)$; and the temperature contributions are $\frac{\partial Q}{\partial T_{I}}=m \times c p$ and $\frac{\partial Q}{\partial T_{O}}=-m \times c p$. So the expression for the uncertainty becomes:

$$
U_{Q}=\sqrt{\left[c p \times\left(T_{I}-T_{O}\right) \times U_{m}\right]^{2}+\left[m \times\left(T_{I}-T_{O}\right) \times U_{c p}\right]^{2}+\left[m \times c p \times U_{T_{I}}\right]^{2}+\left[-m \times c p \times U_{T_{O}}\right]^{2}}
$$

Now, normalizing this equation by dividing all terms by $Q$ in the form of $m \times \operatorname{cp} x\left(T_{I}-T_{O}\right)$,

$$
\frac{U_{Q}}{Q}=\sqrt{\left[\frac{U_{m}}{m}\right]^{2}+\left[\frac{U_{c p}}{c p}\right]^{2}+\left[\frac{U_{T_{I}}}{\left(T_{I}-T_{O}\right)}\right]^{2}+\left[-\frac{U_{T_{O}}}{\left(T_{I}-T_{O}\right)}\right]^{2}}
$$

Finally, a reasonable assumption is that there is negligible uncertainty in a property such as specific heat. Thus $U_{c p}=0$ and Eq. (12) can be written as:

$$
U_{Q}=Q \sqrt{\left[\frac{U_{m}}{m}\right]^{2}+\left[\frac{U_{T_{I}}}{\left(T_{I}-T_{O}\right)}\right]^{2}+\left[-\frac{U_{T_{O}}}{\left(T_{I}-T_{o}\right)}\right]^{2}}
$$

Typically, $U_{Q} / Q$ ought to be less than $0.15(15 \%)$ for each fluid side but at this point a fixed maximum uncertainty should not be established since it is dependent upon the available margin or excess capacity of the cooler or heat exchanger. If an exchanger has a large performance margin (excess heat removal capacity), larger uncertainties can be tolerated. But certainly any unexpectedly large deviation(s) on the hot or cold fluid side(s) can be cause for caution. 
To calculate the overall heat transfer coefficient, a single value for the heat transfer rate must be employed. Theoretically, the heat transferred from the hot side is equal to the gain of the cold side, or $Q=Q_{h}=Q_{c}$. In practice however, the measured values will differ depending on measurement uncertainties. The heat balance error (HBE), between the hot side and cold side, is determined by:

$$
\operatorname{HBE}(\%)=100 \% \times \frac{Q_{h}-Q_{c}}{Q_{h}}
$$

As long as HBE is not equal to zero, the hot side load will never exactly equal the cold side load so that a composite heat load is computed from:

$$
\mathrm{Q}=\frac{Q_{c} U_{Q_{h}}^{2}+Q_{h} U_{Q_{c}}^{2}}{U_{Q_{h}}^{2}+U_{Q_{c}}^{2}}
$$

The associated uncertainty in this composite heat balance is:

$$
U_{H B E}=100 \% \times \frac{Q_{h}}{Q_{c}} \sqrt{\left(\frac{U_{m_{h}}}{m_{h}}\right)^{2}+\left(\frac{U_{T_{h I}}}{T_{h I}-T_{h O}}\right)^{2}+\left(\frac{-U_{T_{h O}}}{T_{h I}-T_{h O}}\right)^{2}+\left(\frac{U_{m_{c}}}{m_{c}}\right)^{2}+\left(\frac{U_{T_{c l}}}{T_{c O}-T_{c l}}\right)^{2}+\left(\frac{-U_{T_{c O}}}{T_{c O}-T_{c l}}\right)^{2}}
$$

If $|H B E|<\left|U_{H B E}\right|$, then the test results and predicted test measurement uncertainties are validated and the test overall heat transfer coefficient may be found. By the same propagation-of-error method to establish uncertainty in heat transfer rates, an uncertainty in the test overall heat transfer coefficient may be obtained and compared or extrapolated to design basis conditions. Note that this procedure can be followed as a pretest with estimated parameters to justify the actual testing. If uncertainty levels are forecast to be too high, the testing may be postponed.

Next is a sample calculation applied to shell-and-tube exchanger data, taken at steady-state conditions, and indicates that a majority of the temperature uncertainty is due to the inherent nature of the probe/DAS system rather than due to any sampling or averaging bias. Given the following shell-and-tube heat exchanger information, the test uncertainty may be calculated; the fluid is water on both sides:

- differential temperature on the shellside (hot side) $=12.8^{\circ} \mathrm{C}$

- differential temperature on the tubeside (cold side) $=21.7{ }^{\circ} \mathrm{C}$

- $\quad$ shellside flow $=30,283 \mathrm{~L} / \mathrm{min}$

- tubeside flow $=17,034 \mathrm{~L} / \mathrm{min}$

- heat transfer rate for the shellside $=26,962 \mathrm{~kW}$

- heat transfer rate for the tubeside $=25,716 \mathrm{~kW}$

- shellside temperature uncertainty (only for the probe/DAS) $=+/-0.56^{\circ} \mathrm{C}$

- tubeside temperature uncertainty (only for the probe/DAS) $=+/-0.56{ }^{\circ} \mathrm{C}$

- shellside flow instrument inaccuracy $=+/-1514 \mathrm{~L} / \mathrm{min}$

- tubeside flow instrument inaccuracy $=+/-1136 \mathrm{~L} / \mathrm{min}$

Neglecting density differences and since $U_{m} / m$ is dimensionless, volumetric flow parameters may be used instead of mass and the uncertainty for the shellside heat load is:

$$
U_{Q_{h}}=26,962 \mathrm{~kW} \sqrt{\left[\frac{1514}{30,283}\right]^{2}+\left[\frac{0.56}{12.8}\right]^{2}+\left[-\frac{0.56}{12.8}\right]^{2}}=2145 \mathrm{~kW}
$$

Similarly for the tubeside,

$$
U_{Q_{c}}=25,716 \mathrm{~kW} \sqrt{\left[\frac{1136}{17,034}\right]^{2}+\left[\frac{0.56}{21.7}\right]^{2}+\left[-\frac{0.56}{21.7}\right]^{2}}=1955 \mathrm{~kW}
$$

These individual load uncertainties, $\frac{U_{Q_{h}}}{Q_{h}}=0.080(8.0 \%)$ and $\frac{U_{Q_{c}}}{Q_{c}}=0.076(7.6 \%)$, are reasonable and therefore the best value 
for the heat rate in evaluating the overall heat transfer coefficient is:

$$
\mathrm{Q}=\frac{25,716(2145)^{2}+26,962(1955)^{2}}{2145^{2}+1955^{2}}=26,281 \mathrm{~kW}
$$

Also, the heat balance error may be found:

$$
\operatorname{HBE}(\%)=100 \% \times \frac{26,962-25,716}{26,962}=4.6 \%
$$

while the composite load uncertainty is:

$$
U_{H B E}=100 \% \times \frac{26,962}{25,716} \sqrt{\left(\frac{1514}{30,283}\right)^{2}+\left(\frac{0.56}{12.8}\right)^{2}+\left(\frac{-0.56}{12.8}\right)^{2}+\left(\frac{1136}{17,034}\right)^{2}+\left(\frac{0.56}{21.7}\right)^{2}+\left(\frac{-0.56}{21.7}\right)^{2}}=11.5 \%
$$

This indicates that there must be at least an $11.5 \%$ margin at the time of any testing. In terms of heat transfer rate, the rate calculated from nominal test values (before uncertainties) must exceed the acceptance criterion heat transfer rate by $11.5 \%$. This result is based on $+/-0.56{ }^{\circ} \mathrm{C}$ for each of the temperatures, which only accounts for a probe/DAS uncertainty. Furthermore, since $|H B E|<\left|U_{H B E}\right|$ or $|4.6 \%|<|11.5 \%|$ then it is likely that the measurements are valid.

To assess the impact of including temporal and spatial uncertainties as demonstrated to be $+/-0.5625{ }^{\circ} \mathrm{C}$ from Eq. (6) in the prior sample calculation, this value is taken to apply to all four temperatures replacing the DAS-alone value of $+/-0.56^{\circ} \mathrm{C}$ :

$$
U_{H B E}=100 \% \times \frac{26,962}{25,716} \sqrt{\left(\frac{1514}{30,283}\right)^{2}+\left(\frac{0.5625}{12.8}\right)^{2}+\left(\frac{-0.5625}{12.8}\right)^{2}+\left(\frac{1136}{17,034}\right)^{2}+\left(\frac{0.5625}{21.7}\right)^{2}+\left(\frac{-0.5625}{21.7}\right)^{2}}=11.6 \%
$$

So the best determination of $Q$ is $26,281+/-3049 \mathrm{~kW}$ or $29,330 \mathrm{~kW} \geq Q \geq 23,232 \mathrm{~kW}$. For the most conservative approach, the overall heat transfer coefficient is computed from the lower limit of $23,232 \mathrm{~kW}$ as this is the exchanger's predicted worst-case performance when measurement, DAS, and other uncertainties are taken into account. If such information is obtained during a pretest and if the predicted performance of the cooler is extrapolated to fall below design but it's reasoned that the deficiency is due to measurement accuracy rather than fouling, then the testing may be postponed until flow conditions favor lower uncertainties, or more accurate DAS components might be employed. The final result for this case, including temporal and spatial deviations, indicates that a majority of the temperature uncertainty is due to the inherent nature of the probe/DAS system rather than due to any sampling or averaging bias. Note that if all the temperature uncertainties were equal to zero, $U_{H B E}$ reduces to $8.7 \%$ indicating that temperature measurements are a significant source of uncertainty, as are flow measurements. It also reinforces the need to carefully consider uncertainty in temperature measurements even prior to taking data.

\section{Conclusions}

An approach has been formulated utilizing a propagation-of-error method to compute an uncertainty level for heat exchanger load. Instrument type and quantity, error, and statistical variations all contribute to temperature uncertainty. Based on standard deviations, confidence levels, and Student t-distribution values, a series of sample calculations has established the effects of the temperature parameters. The methodology is simple and practical and allows data assessment for differing sampling times, number of sensors, and sensor accuracy but does require data from steady-state conditions. The validity of the sample heat exchanger calculation is reinforced as the results are reasonable when compared to other studies. For instance, Wu and Vierow (2006) instrumented the condenser tube of a double-pipe heat exchanger using thermocouple probes having an inherent uncertainty of $+/$ $0.70{ }^{\circ} \mathrm{C}$. Flows were established through reservoir level readings with errors of $+/-2 \%$ and $+/-1 \%$ for the hot side and cold side, respectively. This combination of temperature and flow accuracies led to an uncertainty in the overall heat transfer rate of $9.5 \%$ at the highest heat load. Likewise, the performance of a vertical, U-shaped ground heat exchanger was determined using thermistors and thermocouples, $+/-0.10{ }^{\circ} \mathrm{C}$, and flow measured at $+/-2 \%$ (Sharqawy et al., 2009). All uncertainties included sensor performance, calibration, and data acquisition effects. This level of instrument variance yielded a total uncertainty of $5.0 \%$ in the heat transfer rate of the ground exchanger. In an effort to reduce the size of heat exchangers, Chowdhuri et al. (2011) investigated the use of rod inserts for heat transfer tubes; air flow was measured by a pitot tube and temperatures by type-K thermocouples. An analysis of uncertainties resulted in $1.8 \%$ and $8.2 \%$ for the air flow and heat transfer, respectively. 


\section{Nomenclature}

$B \quad$ bias

$\quad$ specific heat $\left(\mathrm{kJ} /\left(\mathrm{kg}^{\circ} \mathrm{C}\right)\right)$

$J \quad$ number of uncertainty sources

$m \quad$ mass flow rate $(\mathrm{kg} / \mathrm{h})$

$N \quad$ number of measurements

$Q \quad$ heat load $(\mathrm{kW})$

$R \quad$ any heat exchanger parameter

$S \quad$ sample standard deviation

$t \quad$ Student $t$-distribution value

$T \quad$ temperature $\left({ }^{\circ} \mathrm{C}\right)$

$U \quad$ uncertainty

$X \quad$ data point, measurement

$Z \quad$ independent variable

$\theta \quad$ sensitivity coefficient

$\sigma \quad$ standard deviation

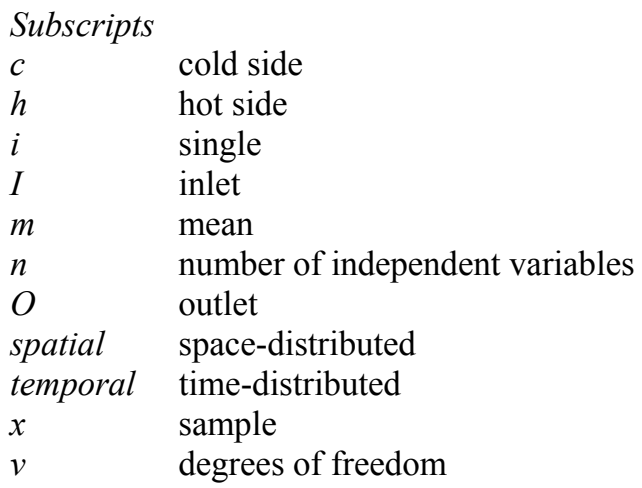

\section{References}

ASME PTC 19.3. 1974. Temperature measurement. American Society of Mechanical Engineers Report, New York, NY. ASME PTC 19.3 TW-2010. 2010. Thermowells. American Society of Mechanical Engineers Report, New York, NY.

Badar M.A., Zubair S.M., and Sheikh A.K. 1993. Uncertainty analysis of heat-exchanger thermal designs using the monte carlo simulation technique. Energy, Vol. 18, No. 8, pp. 859-866.

Cho S.M. 1987. Uncertainty analysis of heat exchanger thermal-hydraulic designs. Heat Transfer Eng., Vol. 8, No. 2, pp. 63-74.

Chowdhuri M.A.K., Hossain R.A., and Sarkar M.A.R. 2011. An experimental investigation of turbulent flow heat transfer through tube with rod-pin insert. International Journal of Engineering, Science and Technology, Vol. 3, No. 4, pp. 76-81.

Coleman H.W. and Steele W.G. 1999. Experimentation and uncertainty analysis for engineers. John Wiley and Sons Inc., NY. EPRI TR-107397. 1998. Service water heat exchanger testing guidelines. Electric Power Research Institute Report, Palo Alto, CA. James C.A., Taylor R.P., and Hodge B.K. 1995. The application of uncertainty analysis to cross-flow heat exchanger performance predictions. Heat Transfer Eng., Vol. 16, No. 4, pp. 50-62.

Lewis T. and Phillips D. 1993. Uncertainty analyses of heat exchanger performance. 1993 International Joint Power Generation Conference, Kansas City, MO, PWR, Vol. 22, pp. 121-127.

Prasad R.C., Karmeshu F., and Bharadwaj K.K. 2002. Stochastic modeling of heat exchanger response to data uncertainties. Appl. Math. Modelling, Vol. 26, pp. 715-726.

Sharqawy M.H., Mokheimer E.M., Habib M.A., Badr H.M., Said S.A., and Al-Shayea N.A. 2009. Energy, exergy and uncertainty analyses of the thermal response test for a ground heat exchanger. Int. J. Energy Res., Vol. 33, pp. 582-592.

Wu T. and Vierow, K. 2006. A local heat flux measurement technique for inclined heat exchanger tubes. Exp. Heat Transfer, Vol. 19, pp. 1-14.

Biographical notes

R.A. Tatara received his B.S.Ch.E., M.S., and Ph.D. in chemical engineering from Northwestern University and has over twenty-five years experience in heat exchangers and cooling water systems. Currently, he is designing and testing solar energy collectors for crop drying and is an associate professor with the Department of Technology, Northern Illinois University, DeKalb, Illinois, USA. 
G.M. Lupia was a senior technical specialist at CSI Technologies, Inc., concentrating in raw-water cooling systems analysis as well as general corrosion issues for power plants. He holds a BSME from the Illinois Institute of Technology and currently is a senior engineer specializing in heat exchangers, cooling water systems, and buried pipe corrosion with Exelon Generation Company, LLC., Warrenville, Illinois, USA.

Received February 2011

Accepted September 2011

Final acceptance in revised form September 2011 